\title{
Fasting Blood Glucose Profile among Secondary School Adolescents in Ado-Ekiti, Nigeria
}

\author{
I. O. Oluwayemi, ${ }^{1}$ S. J. Brink, ${ }^{2}$ E. E. Oyenusi, ${ }^{3}$ O. A. Oduwole, ${ }^{3}$ and M. A. Oluwayemi ${ }^{4}$ \\ ${ }^{1}$ Department of Paediatrics, College of Medicine, Ekiti State University, Ado-Ekiti, Ekiti State, Nigeria \\ ${ }^{2}$ New England Diabetes and Endocrine Center, USA \\ ${ }^{3}$ Paediatric Endocrinology Training Centre for West Africa, Lagos University Teaching Hospital, Idi-Araba, Lagos, Nigeria \\ ${ }^{4}$ Clinical Nursing Services, Ekiti State University Teaching Hospital, Ado-Ekiti, Ekiti State, Nigeria
}

Correspondence should be addressed to I. O. Oluwayemi; dareoluwayemi@gmail.com

Received 17 December 2014; Revised 6 March 2015; Accepted 21 March 2015

Academic Editor: Pedro Moreira

Copyright (C) 2015 I. O. Oluwayemi et al. This is an open access article distributed under the Creative Commons Attribution License, which permits unrestricted use, distribution, and reproduction in any medium, provided the original work is properly cited.

\begin{abstract}
Background. Over the past two decades there has been an increase in type 2 diabetes mellitus (T2DM) in children. Baseline data is needed to assess the impact of changing lifestyles on Ado-Ekiti, a previously semiurban community in Southwest Nigeria. This study was therefore conducted to assess the fasting blood glucose (FBG) of adolescents in Ado-Ekiti, Nigeria. Methodology. This was a cross-sectional study involving 628 adolescents from three different secondary schools in Ado-Ekiti, Nigeria. With parental consent, volunteers completed a structured questionnaire, and an overnight FBG was measured. Results. There were 346 males and 282 females (male : female ratio $=1.2: 1$ ). Their ages ranged from 10 to 19 years (mean age: $14.2 \pm 1.7$ years). Four hundred and fortyfour $(70.7 \%)$ had normal FBG, while $180(28.7 \%)$ and 4 (0.6\%) had FBG in the prediabetic and diabetic range, respectively. Female gender, age group 10-14 years, and family history of obesity were significantly associated with impaired FBG $(P$ value $<0.001$, $<0.001$, and 0.045 , resp.). Conclusion. Impaired FBG is common among secondary school adolescents and it is more prevalent among younger female adolescents (10-14 years) with positive family history of obesity.
\end{abstract}

\section{Introduction}

There is rising incidence of noncommunicable diseases like diabetes, hypertension, and coronary heart diseases globally [1-3]. Increased risk of impaired glucose tolerance, insulin resistance, and type 2 diabetes (T2DM) has been found to be associated with obesity in adolescents [4]. The American Academy of Paediatrics and the American Diabetes Association have recommended that children aged 10 years or at the onset of puberty who are overweight and have at least two other risk factors should be tested every two years for T2DM $[5,6]$. The risk factors for developing T2DM include family history of T2DM in first- or second-degree relative, belonging to certain ethnic groups (i.e., Native American, African American, Hispanic, Japanese, or other Asian/Pacific Islanders), or having signs associated with insulin resistance (hypertension, dyslipidemia, acanthosis nigricans, or polycystic ovarian syndrome) $[5,7]$. Similarly other endogenous populations, for instance, in Canada, Australia, Russia, and Latin America, may share similar genetic predispositions [8].

Beck-Nielsen and Groop [9] proposed a three-stage model for the development of T2DM. Stage 1 includes fasting hyperinsulinemia with normal or slightly increased blood glucose, especially mild fasting hyperglycemia. Stage 2 is characterized by prediabetic glucose intolerance with insulin resistance, and Stage 3 is development of classical symptomatic or nonsymptomatic T2DM with more persistent hyperglycemia present. Many of the macrovascular changes associated with diabetes and related to cardiovascular disease (CVD) begin in Stages 1 and 2, well before overt diagnosis [10].

Adolescents are a dependent population and development of diabetes mellitus or other noncommunicable diseases will pose a burden to parents and society at large,, hence the need to continually assess the FBG of adolescents in rapidly changing communities. This will help in early 
TABLE 1: Fasting blood glucose grading according to age group.

\begin{tabular}{lcccr}
\hline & & Fasting blood glucose (FBG) grading & & \\
Age group & Euglycemia & Impaired FBG (prediabetic) & Diabetes FBG \\
& $2.8-5.5 \mathrm{mmol} / \mathrm{L}$ & $5.6-6.9 \mathrm{mmol} / \mathrm{L}$ & $\geq 7 \mathrm{mmol} / \mathrm{L}$ & Total \\
& $(50-99 \mathrm{mg} / \mathrm{dL})$ & $(100-125 \mathrm{mg} / \mathrm{dL})$ & 4 & \\
\hline $10-14$ years & 232 & 121 & 0 & 357 \\
$15-19$ years & 212 & 59 & $4(0.6)$ & $628(100)$ \\
\hline Total $(\%)$ & $444(70.7)$ & $180(28.7)$ & $0 . \mathrm{mL})$ & \\
\hline
\end{tabular}

detection and hopefully the control of the prediabetic phase through education and lifestyle modification. It will also help in prompt commencement of treatment in those with established diabetes to improve quality of life and prevent complications. Data on FBG among Nigerian adolescents are scarce, hence the need for the present study especially in Ado-Ekiti, a relatively new and rapidly developing state capital in Nigeria with changes in lifestyle associated with urbanization such as inappropriate dietary practices (fast food consumption, low fruit consumption) and low physical activity $[11,12]$.

\section{Material and Methods}

Secondary school adolescents of both sexes who satisfied the inclusion criteria were recruited for the study from three different schools in Ado-Ekiti, the capital of Ekiti State, Nigeria. Ethical clearance and permission to enter the schools were obtained from the Research and Ethics Committee of the Ekiti State University Teaching Hospital, Ado-Ekiti, and the state's Ministry of Education, respectively. Inclusion criteria for the study were apparently healthy secondary school adolescents aged between 10 and 19 years, with no history of diabetes. With parental consent, volunteers completed a structured questionnaire, and FBG was measured after an overnight fast. Capillary blood sample was collected for FBG measurement using Accu-Chek Active glucometer, after the thumb or index finger had been cleaned with wet (water) cotton wool. Data was entered and analyzed using SPSS 16.0 for Windows (SPSS Inc., Chicago, USA). Subjects were grouped based on their age, gender, family history of obesity and diabetes, and fasting blood glucose (FBG). Cross tabulation and tests for association with chi square $\left(\chi^{2}\right)$ were done and $P$ values less than 0.05 were regarded as significant.

\section{Results}

The subjects in the present study comprised 346 male and 282 female adolescents (male to female ratio of $1.2: 1$ ) with mean age of $14.2 \pm 1.7$ years and age range of 10 to 19 years. Four $(0.6 \%)$ adolescents had FBG in diabetic range; 180 (28.7\%) had impaired FBG and 121 (67\%) of these were in the 10-14year age group. Also, all the four adolescents who had diabetic FBG range were in the same 10-14-year age group (Table 1). There were $77(41.8 \%)$ males and $107(58.2 \%)$ females in the $184(29.3 \%)$ adolescents with high FBG (180 in prediabetic

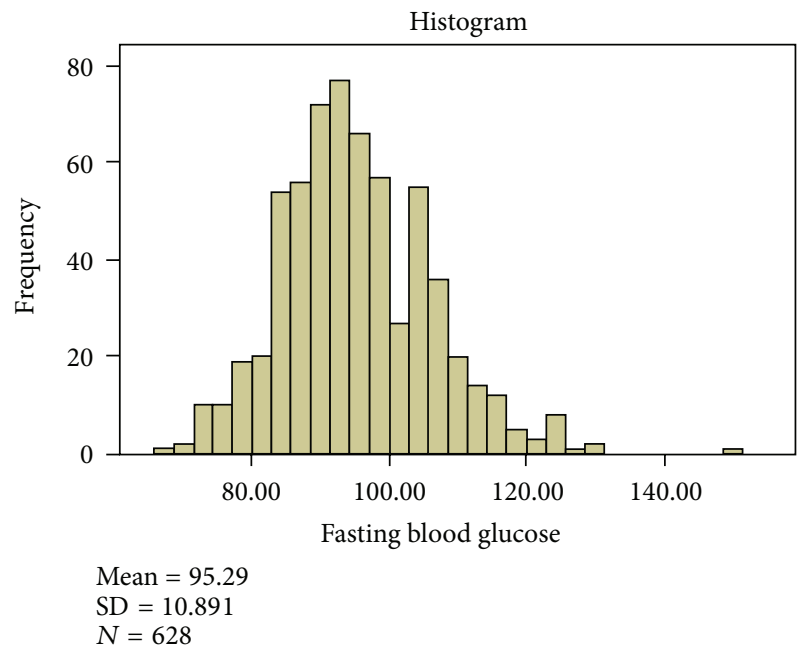

FIGURE 1: Fasting blood glucose (mg/dL) pattern in 628 secondary school adolescents.

and 4 in diabetic range) among the studied 628 adolescents giving a male to female ratio of $0.7: 1$. The proportion of males with high FBG of the overall studied male population of 346 was $77(22.3 \%)$ while the proportion of females with high FBG of the overall female population of 282 was 107 (37.9\%). This difference was statistically significant $\left(\chi^{2}=18.462, \mathrm{df}=\right.$ $1, P=0.000$ ). Figure 1 shows the FBG profile in the studied sample of secondary school adolescents in Ado-Ekiti. The mean (SD) for their FBG was 95.3 (10.9), median was 94.0, and it ranged from 68 to $149 \mathrm{mg} / \mathrm{dL}$. The variability of FBG in both genders was comparable except that the median FBG for females was slightly higher (Figure 2). The mean (SD) FBG for males was 93.4 (10.8) and for females was 97.6 (10.5). The FBG chart for age (Figure 3) showed two distinct peaks which occurred at 12 and 17 years though there appears to be a little rise at the age of 14 years. Female gender, early adolescence (age group 10-14 years), and family history of obesity have statistically significant influence on FBG levels ( $P$ value $<0.001,<0.001$, and 0.045 , resp. $)$.

\section{Discussion}

The review of the fasting blood glucose of the adolescents in Ado-Ekiti showed that the majority $(70.7 \%)$ had normal FBG, and a rather large number, about a third (28.7\%), 


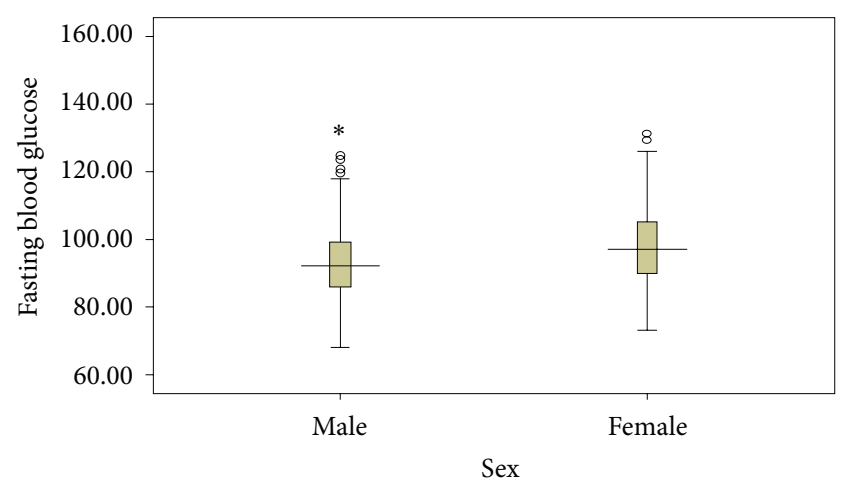

Figure 2: Comparison of FBG (mg/dL) pattern in male and female population.

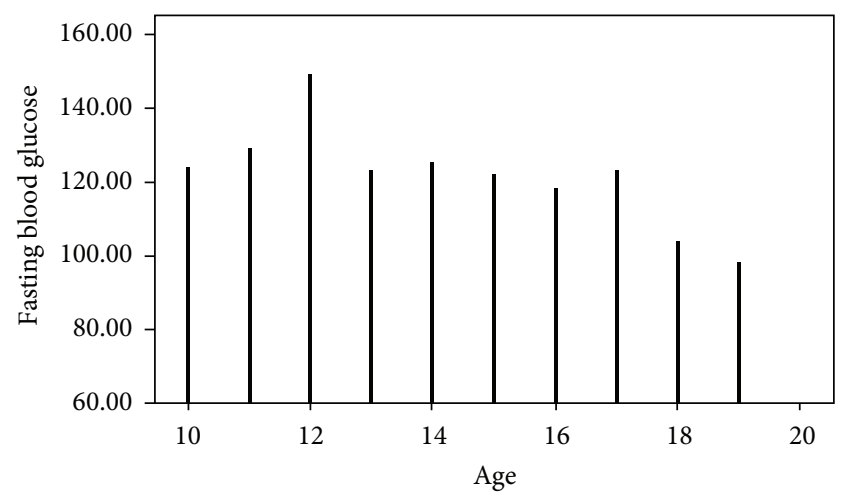

Figure 3: Profile of fasting blood glucose ( $\mathrm{mg} / \mathrm{dL}$ ) according to age (years) of adolescents in Ado-Ekiti.

had impaired FBG in the prediabetic range and $0.6 \%$ of them had presumed diabetic FBG range. The possibility of secondary school adolescents falsely denying taking some juice or snack before the test cannot be completely ruled out and this may contribute to the unusually high proportion of those with impaired FBG in this study. There may also be some bias using plasma meter BG readings rather than more centralized sampling systems as reported in other studies $[2,13]$. Some nervousness of participants not used to finger pricks may also have contributed to some false elevations and repeat testing will be proposed to double-check these results. The FBG profile of the adolescents plotted against their ages showed two distinct peaks which occurred at 12 and 17 years (Figure 3); this is similar to the peaks in their BMI which occurred at the same ages of 12 and 17 years. This finding is in concordance with findings among adolescents in Beijing area of China [14] whose FBG peaked at 12-13 years and at 17 years of age. Increased secretion of growth hormone and adrenocortical and gonadal hormones during puberty usually causes increase in insulin resistance and this could explain the peaks in FBG of adolescents at 12 and 17 years which roughly correspond to early and late phase of puberty in both genders combined [13]. It was also demonstrated in this index study that early adolescence (age group 10-14 years), female sex, and family history of obesity have statistically significant association with impaired fasting blood glucose and this finding agrees with findings from previous studies $[13,14]$. Moreover, the mean FBG in the male and female adolescents in index study $(93.4 \pm 10.8$; $97.6 \pm$ $10.5 \mathrm{mg} / \mathrm{dL}$, resp.) is slightly higher than those of Moroccan [2] male and female adolescents of a similar age (92 \pm 14.0 ; $89 \pm 10.0 \mathrm{mg} / \mathrm{dL}$, resp.). This difference may be due to different methods used for analysis of FBG. In the Moroccan study, Mehdad et al. [2] used hexokinase method to determine FBG while the current study assessed capillary FBG with AccuChek Active glucometer. Also, the Moroccan study [2] had a smaller and disproportionate study population (44 males; 123 females) compared to the index study of 346 male and 282 female adolescents.

\section{Strength and Limitation of the Study}

(1) The sample size for this study is adequate and both genders were well represented.

(2) The population of adolescents available for this study from private secondary school was small compared to those from public secondary schools, hence preventing meaningful comparison.

\section{Conclusion}

The present study among secondary school adolescents in Ado-Ekiti, Nigeria, showed that FBG was normal in twothirds of them while the remaining one-third had impaired FBG. Also, impaired FBG is significantly more common among younger female adolescents (10-14 years) with positive family history of obesity.

\section{Conflict of Interests}

The authors declare that there is no conflict of interests regarding the publication of this paper.

\section{Acknowledgments}

The authors wish to sincerely appreciate all who participated and assisted in making this study a reality. Their special thanks also go to ISPAD (International Society of Paediatric and Adolescent Diabetes) for providing a grant for this study.

\section{References}

[1] World Health Organization, "Obesity and overweight," Fact sheet No 311, 2012.

[2] S. Mehdad, A. Hamrani, K. El Kari et al., "Body mass index, waist circumference, body fat, fasting blood glucose in a sample of Moroccan adolescents aged 11-17 years," Journal of Nutrition and Metabolism, vol. 2012, Article ID 510458, 7 pages, 2012.

[3] A. O. Akinpelu, O. O. Oyewole, and K. S. Oritogun, "Overweight and obesity: does it occur in Nigerian adolescents in an urban community?" International Journal of Biomedical and Healthcare Science, vol. 4, pp. 11-17, 2008.

[4] E. P. Whitlock, S. B. Williams, R. Gold, P. R. Smith, and S. A. Shipman, "Screening and interventions for childhood 
overweight: a summary of evidence for the US Preventive Services Task Force," Pediatrics, vol. 116, no. 1, pp. el25-el44, 2005.

[5] P. W. Speiser, M. C. J. Rudolf, H. Anhalt et al., "Consensus statement: childhood obesity," Journal of Clinical Endocrinology and Metabolism, vol. 90, no. 3, pp. 1871-1887, 2005.

[6] S. Gahagan, J. Silverstein, and Committee on Native American Child Health and Section on Endocrinology, "Prevention and treatment of type 2 diabetes mellitus in children, with special emphasis on American Indian and Alaska Native children," Pediatrics, vol. 112, no. 4, p. e328, 2003.

[7] S. B. Sondike, "Overweight and obesity," in Pediatric Endocrinology and Inborn Error of Metabolism, K. Sarafoglou, G. F. Hoffmann, and K. S. Roth, Eds., pp. 275-293, The McGraw-Hill, 2009.

[8] S. J. Brink, "How should children and youth with type 2 diabetes be treated?" in Theoretical and Practical Update in Paediatric Endocrinology and Diabetes, I. P. Velea, C. Paul, and S. J. Brink, Eds., pp. 89-126, Mirton, Timişoara, Romania, 2014.

[9] H. Beck-Nielsen and L. C. Groop, "Metabolic and genetic characterization of prediabetic states. Sequence of events leading to non-insulin-dependent diabetes mellitus," The Journal of Clinical Investigation, vol. 94, no. 5, pp. 1714-1721, 1994.

[10] A. Jessup and J. S. Harrell, “The metabolic syndrome: look for it in children and adolescents, too!", Clinical Diabetes, vol. 23, no. 1, pp. 26-32, 2005.

[11] A. N. Onyiriuka, D. D. Umoru, and A. N. Ibeawuchi, "Weight status and eating habits of adolescent Nigerian urban secondary school girls," South African Journal of Child Health, vol. 7, no. 3, pp. 108-112, 2013.

[12] A. K. Singh, A. Maheshwari, N. Sharma, and K. Anand, "Lifestyle associated risk factors in adolescents," Indian Journal of Pediatrics, vol. 73, no. 10, pp. 901-906, 2006.

[13] G. Plourde, "Impact of obesity on glucose and lipid profiles in adolescents at different age groups in relation to adulthood," BMC family Ppractice, vol. 3, article 18, 2002.

[14] B.-Y. Cao, J. Mi, C.-X. Gong et al., "Blood glucose profile in children and adolescents in Beijing area," Chinese Journal of Pediatrics, vol. 46, no. 4, pp. 297-300, 2008. 


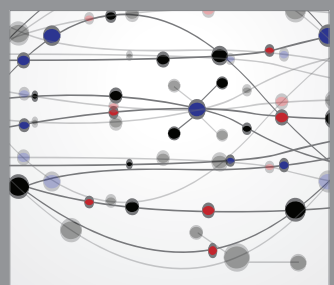

The Scientific World Journal
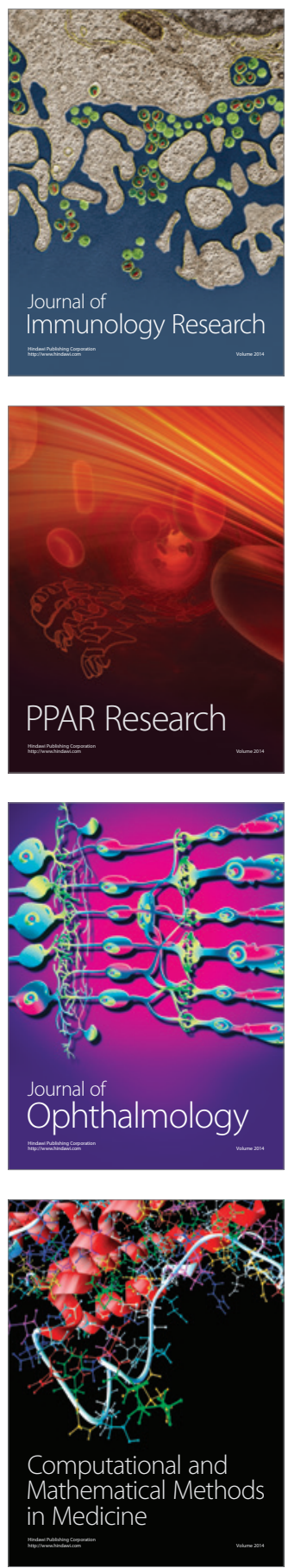

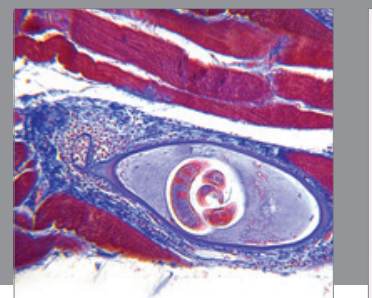

Gastroenterology

Research and Practice
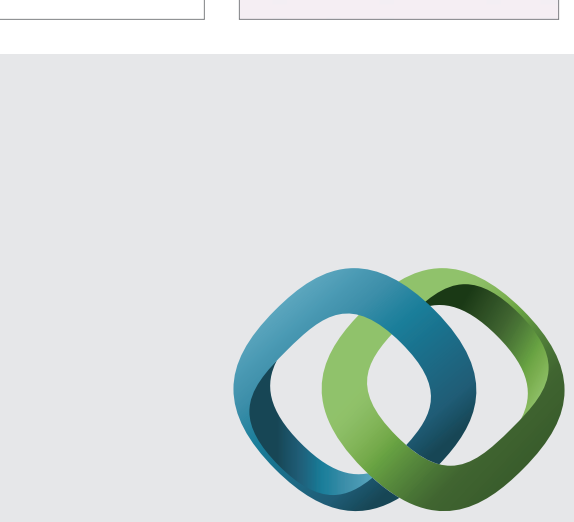

\section{Hindawi}

Submit your manuscripts at

http://www.hindawi.com
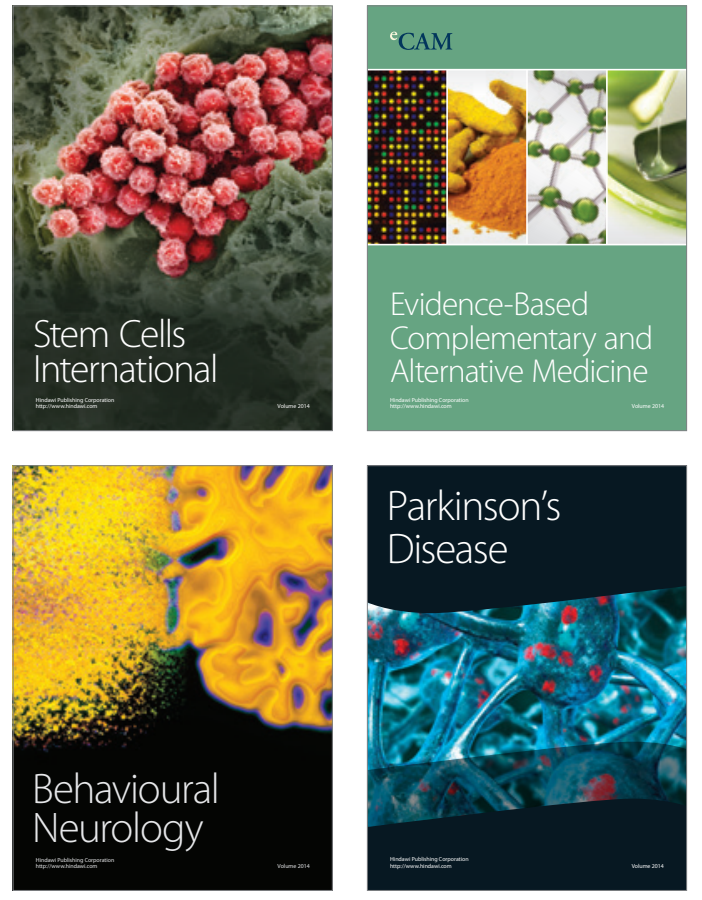
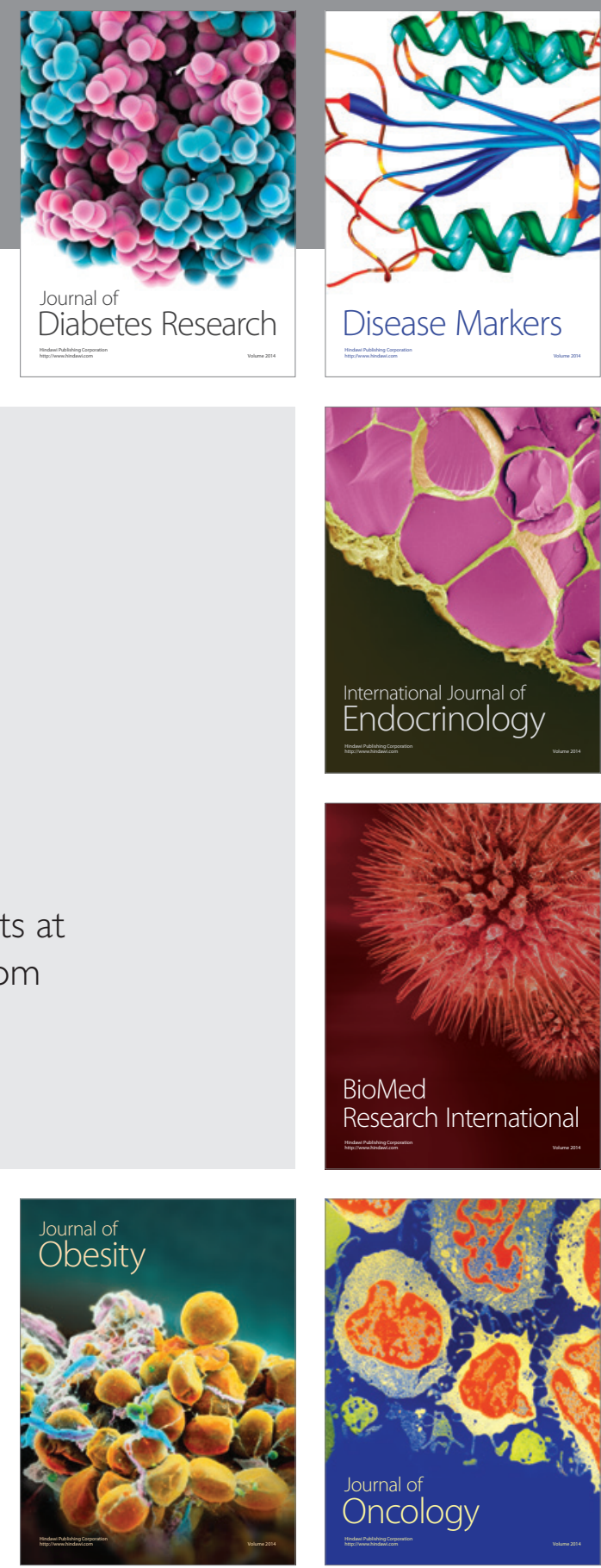

Disease Markers
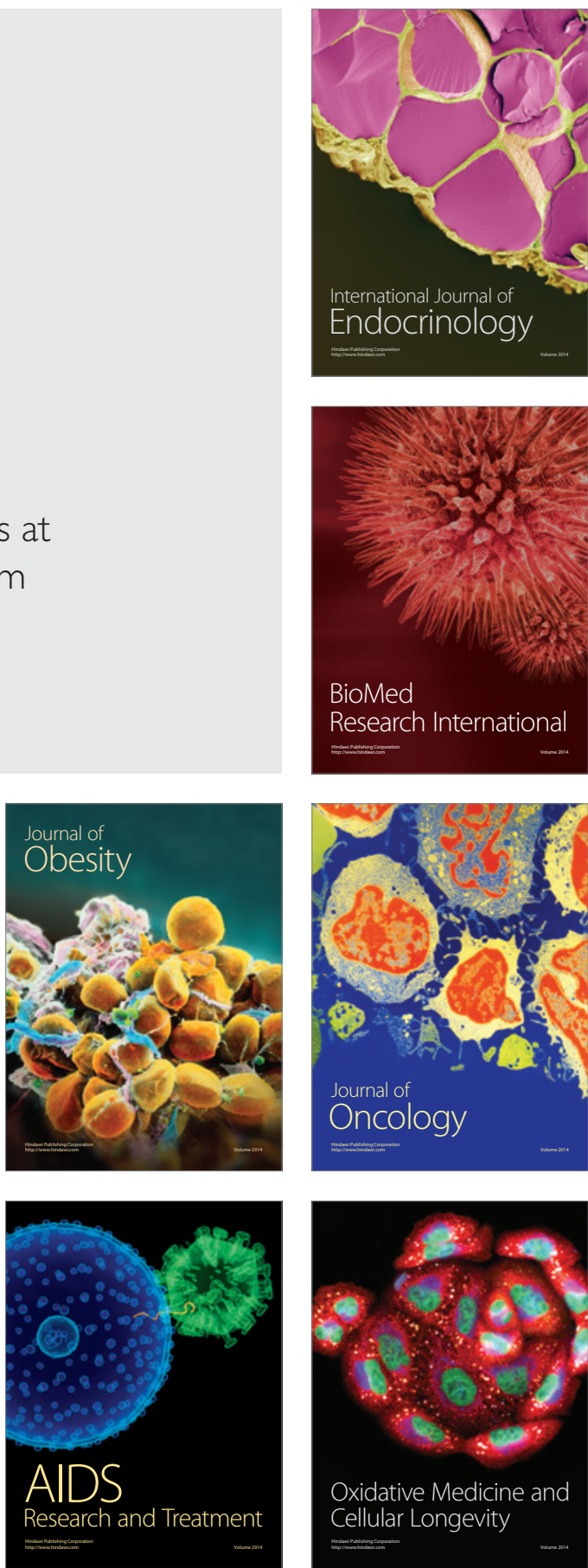